OPEN ACCESS

Edited by:

Raghvendra Mohan Srivastava,

Memorial Sloan Kettering Cancer

Center, United States

Reviewed by:

Avery Dexter Posey Jr.,

University of Pennsylvania,

United States

Rajshekhar Alli,

St. Jude Children's Research Hospital,

United States

*Correspondence:

Rahul Purwa

purwarrahul@iitb.ac.in

†These authors have contributed equally to this work

Specialty section

This article was submitted to

$T$ Cell Biology,

a section of the journal

Frontiers in Immunology

Received: 12 September 2018

Accepted: 27 December 2018

Published: 18 January 2019

Citation:

Dwivedi A, Karulkar A, Ghosh S,

Rafiq A and Purwar R (2019)

Lymphocytes in Cellular Therapy: Functional Regulation of CAR T Cells.

Front. Immunol. 9:3180

doi: 10.3389/fimmu.2018.03180

\section{Lymphocytes in Cellular Therapy: Functional Regulation of CAR T Cells}

\author{
Alka Dwivedi ${ }^{\dagger}$, Atharva Karulkar ${ }^{\dagger}$, Sarbari Ghosh ${ }^{\dagger}$, Afrin Rafiq ${ }^{\dagger}$ and Rahul Purwar ${ }^{*}$ \\ Department of Biosciences and Bioengineering, Indian Institute of Technology Bombay, Mumbai, India
}

Lymphocytes especially autologous T cells have been used for the treatment of numerous indications including cancers, autoimmune disorders and infectious diseases. Very recently, FDA approved Chimeric Antigen Receptor T cells (CAR T cells) therapy for relapse and refractory CD19+ B cell acute lymphoblastic leukemia ( $r / r$ B-ALL) and $r / r$ diffuse large $B$ cell lymphoma ( $r / r$ DLBCL) upon their remarkable success in multiple Phase I-II clinical trials. While CAR T cells are considered as major breakthrough in the field of cancer immunotherapy, the regulation of CAR T cells remains poorly understood. In this review we will discuss the strategies that regulate the CAR T cells efficacy and persistence with focus on roles of different structural component of CAR construct. Different domains of CAR construct, for example, antigen binding domain, hinge, transmembrane, and signaling domain as well as immune-regulatory cytokines have significant impact on CAR T cell efficacy. Finally, this review will highlight the strategies that will promote CAR T cells efficacy and will reduce the toxicity.

Keywords: chimeric antigen receptor, cancer immunotherapy, immunoregulation, anti-tumor efficacy, cytokines

\section{INTRODUCTION}

Chimeric antigen receptor T cells (CAR T cells) have achieved remarkable success in the field of cancer tumor immunotherapy since last decade (1). Promising clinical outcomes were observed in case of hematological malignancies leading to FDA-approval of Kymriah ${ }^{\mathrm{TM}}$ and Yescarta ${ }^{\mathrm{TM}}$ for $\mathrm{r} / \mathrm{r}$ B-ALL in pediatric and young adults and adult patients with large B cell lymphoma, respectively. This has led to a paradigm shift in the field of cancer immunotherapy especially in treatment of some hematological cancers across the globe. The enormous success of CAR T cells in hematological malignancies is attributed to numerous factors, most important being the choice of CD19 expression on all B cells $(2,3)$. Other factors are easy sampling of the tumor and trouble free homing of the T cells to hematologic organs such as blood, bone marrow and the lymph nodes (4). On the contrary, efficacy of CAR T cells targeting solid tumors is still in its infancy due to multiple challenges such as lack of unique tumor associated antigen (TAAs), inefficient $\mathrm{T}$ cell homing to the tumor bed and due to immunosuppressive tumor microenvironment. These factors lead to limited persistence and sub-optimal efficacy of the CAR T cells in solid tumor settings (5). 
CARs consist of four main domains, namely ectodomain for specific target antigen recognition and endodomain that provides costimulatory and activation signals. These two domains are connected by hinge and transmembrane domain (Figure 1). The major advantages of CAR T cells over other cell based therapies are (1) killing of tumor targets in a MHC independent manner and thereby overcoming certain tumor escape mechanisms such as MHC-I down regulation and faulty antigen processing, (2) engineering of multiple anti-tumor immuno-modulators, and (3) targeting wide array of antigens (protein, carbohydrate, and glycolipid).

While CAR $\mathrm{T}$ cells are effective in achieving long-term remission in certain types of malignancies, the major challenge remains in controlling CAR $\mathrm{T}$ cells in case of dysregulated activation. Hence, our major focus in this review is to elucidate the structure-function relationship of different components of CAR construct on CAR T cells persistence and efficacy.

\section{Impact of ScFv Ectodomain on CAR T Cell Functions}

The scFv ectodomain, which plays important role in the efficacy and safety of CAR $\mathrm{T}$ cells, is a smallest synthetic functional module containing variable heavy $(\mathrm{VH})$ as well as variable light (VL) chain portion of an antibody linked with a long flexible linker. Most commonly used linker in several CAR construct is (Gly4Ser) $)_{3}$. Glycine residues provide flexibility and serine residues provide solubility. This renders a properly folded scFv thereby maintaining antigen-binding capability of the parental IgG. Differences in orientation of the VH, Linker and VL may affect the scFv's affinity and specificity (6). scFvs are critical for antigen specific CAR T cell activation. Non-specific or crossreactive CARs can augment the off-target toxicity and inefficient CAR T cell activation. Hence, considerable efforts are needed for $\mathrm{scFv}$ designing and its characterization.

Two approaches especially animal immunization and surface display methods have been widely used for designing and screening of scFv. Although the most effective method is immunization, in case of well-conserved antigen there has been reports of generation of neutralizing antibodies due to cross reactivity (7).

There are various antibody display technologies such as phage display, cell display, ribosomal display, mRNA display and DNA display, out of which, phage display is one of the commonly used screening method (8-10). Display methodologies such as phage display overcomes the limitation of immune tolerance since they are based on in vitro selection from naïve or immune libraries (11). Yeast surface display emerged as an alternative technology to phage display, generating $10^{8}-10^{9}$ library members. These antibodies have better affinity and specificity profiles through combination of library screening by flowcytometry and affinity maturation by in vitro codon variation or mating mediated chain shuffling $(12,13)$. In recent years high throughput eukaryotic cell display technologies have been successfully utilized. The advantage of this technology is real time analysis and characterization of library along with machineries for proper folding before being displayed on the surface of the cell. High throughput display technologies creates antibody libraries from which antibody fragments or domains can be selected for better effector function, tissue penetration and pharmacokinetics (14). Therefore, in order to cater the screening of antigen binding of $\mathrm{scFv}$ domains in CAR, either of the above methods have been utilized and have a significant role in deciding the CAR T cell efficacy.

The four important characteristics of $\mathrm{scFv}$ are immunogenicity, affinity, specificity, and its binding epitope. The monoclonal antibodies (mAbs) obtained from murine hybridomas were found to be immunonogenic in humans which resulted in low efficacy and immediate elimination from circulation $(15,16)$. They also showed systemic inflammatory responses resulting in serious physiological complications. Hence humanization of $s c F v$ can help to enhance safety and therapeutic potential of a CAR. Anti-folate receptor $\alpha(\mathrm{FR} \alpha)$ CAR T cells were developed against metastatic ovarian cancer using MOv18scFv which is a murine $\mathrm{mAb}$ for $\mathrm{FR} \alpha$. But, the CAR $\mathrm{T}$ cells showed poor persistence and anti-tumor efficacy $(15,17)$. In another study involving mesothelin-targeted CAR $\mathrm{T}$ cells containing SS1 (murine scFv), anaphylactic shock was observed in a patient. This was probably promoted by IgE antibodies specific for murine scFvs. This further indicates potential immunogenicity of murine $\mathrm{scFv}$ containing CARs (16). These CARs showed less in vivo persistence along with poor anti-tumor efficacy. Less immunogenicity was observed due to humanization resulting in enhanced persistence and safety of CAR T cells. A low affinity but highly specific CAR for epidermal growth factor receptor variant III (EGFRvIII) was humanized and included in the second-generation CAR T cells containing EGFRvIII scFv, 4-1BB and CD3 $\zeta$ domains. Patients infused with this CAR showed minimum off-target toxicity and decreased cytokine release syndrome (18). The above humanized CARs showed better persistence and functionality but they still pose a risk of off-tumor toxicity owing to the $5 \%$ residual mouse sequences. This leads to the necessity of developing fully humanized scFvs, either from phage display or transgenic mouse models. In this connection, M28z CAR, consisting of m912 scFv (fully human anti-mesothelin $\mathrm{mAb}$ ) was generated to resolve the immunogenicity issue which resulted in long term complete remission as reported in in vivo tumor models (19). Few other humanized CARs such as anti-FR $\alpha$ CAR for ovarian cancer and anti-CD22 CAR derived from $\mathrm{m} 971$ are in clinical trials $(2,3,20,21)$. With these advantages of using humanized scFv derived CARs, a case report of anti-HER2 CAR T cells containing $\mathrm{scFv}$ from trastuzumab (humanized mAb-herceptin) showed exceptional fatality with dosage of $1 \times 10^{10}$ cells/infusion (22). In contrast to this, the patients receiving a low dose $\left(1 \times 10^{8}\right.$ cells $/ \mathrm{m}^{2}$ ) of anti-HER2 CAR T cells derived from murine clone FRP5 showed increased tolerance along with minimum toxicity (23). In response to this observation, the change in epitope binding affinity and avidity might have an impact on the efficacy and toxicity of the anti-HER2 CAR T cells. The epitope of HER-2 recognition is distinct for trastuzumab (derived from 4D5 clone) in comparison to murine FRP5 clone. Other factors which might have role in reduced toxicity are the $\mathrm{T}$ cells dosage, lack of lymphodepletion regime and less persistence of murine FRP5 


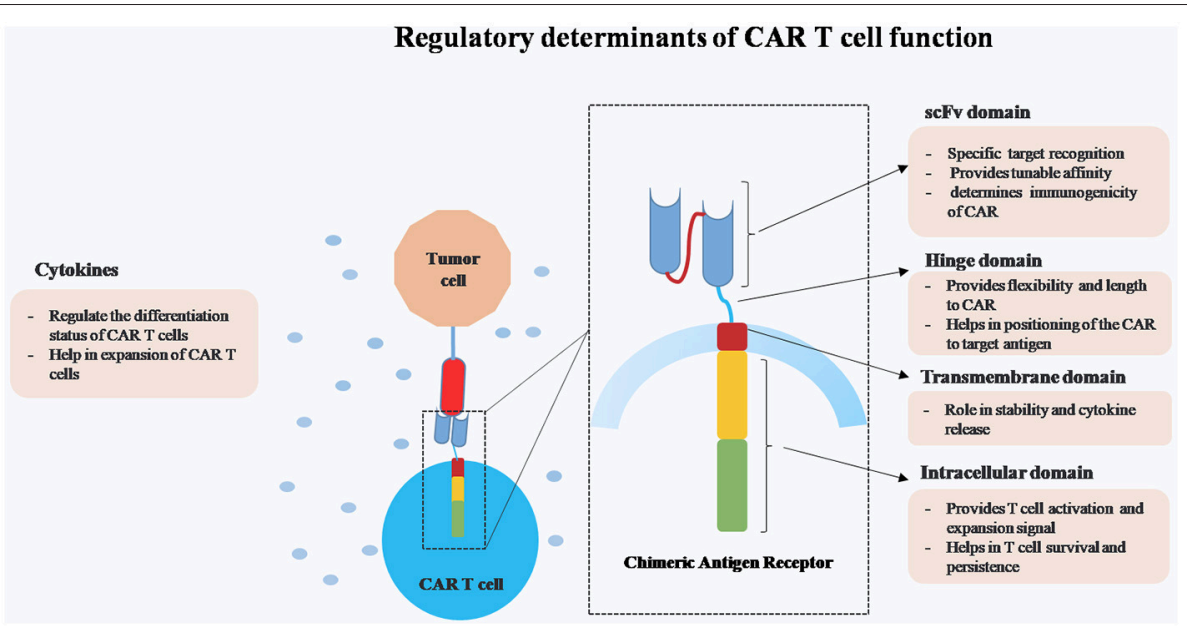

FIGURE 1 | Schematic representation of different regulatory components of Chimeric Antigen Receptor (CAR) T cells.

anti-HER-2 CAR over trastuzumab containing CAR T cells. However, due to involvement of multiple factors in treatment, it remains unclear to identify the exact reason of the exceptional fatality using humanized scFv. Tumor associated antigens (TAAs) are the prominent targets for immunotherapy that are highly expressed on tumor tissue and also expressed at lower level in healthy tissues. This leads to unwanted recognition and sometimes life threatening toxicity. Therefore, the scFv selection is crucial while designing CARs in order to discriminate between tumor cells and normal tissues. To avoid these complications, the interactions between CAR scFvs and target antigens should be carefully understood and a cross reactivity study should be performed before finalization of $s c F v$. One approach is to increase the $s c F v$ affinity toward target antigens. For example, CARs containing high affinity scFvs for receptor tyrosine kinase like orphan receptor I (ROR1) and folate receptor $\beta$ (FR $\beta$ ) have shown superior effector functions than CARs with low affinity scFvs $(24,25)$.

In addition, a fine balance between specificity and affinity for the target antigen also plays a determining role in CAR $\mathrm{T}$ cell function. Affinity tuned CARs having more specificity and less affinity showed high therapeutic index (26). Further, epitope positioning also determines the efficiency of CAR T cell activation. For example, scFv recognizing a CD22 epitope, lying proximal to $\mathrm{B}$ cell plasma membrane showed enhanced anti-tumor function as compared to one recognizing membranedistal epitope (27). Therefore, the approach of incorporating a flexible linker in the CAR construct can modulate the antigen binding.

Upon the long term follow-up of the $\mathrm{r} / \mathrm{r}$ B-ALL patients, it was observed that the tumors have acquired resistance to anti-CD19 CAR $\mathrm{T}$ cells (28-30). Possible reasons for the resistance toward anti-CD19 CAR T cell could be: (1) loss of target antigen, (2) exhaustion and lesser persistence of anti-CD19 CAR $\mathrm{T}$ cells (3) immunosuppressive tumor microenvironment $(31,32)$. To overcome the loss of antigen target due to mono-antigen specific CARs, bi-specific CARs have been designed to recognize two antigens in a true Boolean OR-gate fashion (i.e., either of the two antigens binding should be sufficient to trigger robust $\mathrm{T}$-cell output). The studies have shown that in a high-disease burden setting, the bispecific CD19-CD20 CARs CAR proved both effective and less toxic than single CARs in pre-clinical settings $(33,34)$ Similarly, bispecific CAR has been designed to target both human epidermal growth receptor 2 (HER2) and IL13R $\alpha 2$ and it showed enhanced potency and anti-tumor activity in vivo compared to two separate CARs (35). There are multiple on-going clinical trials in children and adults using bispecific CAR T cells (NCT03241940, NCT03233854, and NCT03448393). As reported in Phase 1 trial of anti-CD19/CD22 CAR T cells, anti-leukemic activity was observed with complete remission in 5/5 patients with CD19 dim/neg B-ALL for a duration of 6 months (20). Long term follow-up of these clinical trials will give the insights on superiority of bispecific CAR strategies over current monospecific CAR T cells.

Hence, these four aspects of $\mathrm{scFv}$ (immunogenicity, specificity, affinity, target positioning) are vital in determining the safety and functional efficacy of CAR T cells.

\section{Regulation by Hinge Region}

Hinge region connects the ectodomain and the transmembrane domain of a CAR. Amino acid fragments from CD $8 \alpha$, IgG1, and IgG4 are the most common hinge used in majority of the CARs. Hinge region can affect the CAR function by providing flexibility and length to a CAR. Function of hinge region may vary depending on the targeted antigen. For example, hinge-less CARs against different antigens like CD19, carcinoembryonic antigen (CEA), neural cell adhesion molecule (NCAM) and 5T4 showed different effector functions compared to CARs having $\mathrm{CH} 2 \mathrm{CH} 3$ hinge adapted from IgG1. CAR T cells against CD19 and 5T4 showed enhanced functional efficacy after adding $\mathrm{CH} 2 \mathrm{CH} 3$ hinge in contrast to CEA and NCAM CAR T cells (36). As described in $\mathrm{scFv}$ section accessibility of a target antigen can modulate the efficacy of a CAR provided by a flexible linker as well as hinge region. Investigators have seen that in case of anti-CD22 
CAR having a spacer derived from IgG1 Fc receptor impacts the positions of the targeted antigen with respect to cell surface and leads to increased efficacy (27). Moreover, length of hinge region is also critical for CAR T cells efficacy, shorter hinge region showed enhanced antitumor efficacy against ROR1 antigen compared with longer hinge (37). Very recently mesothelin CAR containing IgG4 hinge have shown higher efficacy and CAR $\mathrm{T}$ cell proliferation than the CAR without hinge region by bringing the mesothelin antigen proximal to membrane and thereby reducing the steric inhibitory effects between $\mathrm{scFv}$ and its target epitope (38). Recently investigators have found that replacing IgG1 hinge to IgG2 enhanced the efficacy further and reduced off target effect of a CAR against an antigen prostate stem cell antigen (PSCA) which is overexpressed on many solid tumors (39). These findings suggest that hinge region provides the flexibility to overcome the steric hindrances as well as reduces the distance between CAR scFv and antigen. Further studies are ongoing to understand the effect of hinge domain on flexibility, distance and elimination of the off target effects in relation to CARs.

\section{Roles of Transmembrane Domain of CAR Construct in CAR T Cell Function}

Transmembrane region is a linker between hinge region and endodomain of a CAR. Type I proteins such as CD3 $\zeta$, CD28, and $\operatorname{CD} 8 \alpha$ have been used as transmembrane domains in CAR constructs. Earlier it was thought that transmembrane domain does not have much impact on CAR T cell efficacy except anchoring CAR molecule to the membrane. But recent investigations have shown that transmembrane domain impacts the efficacy as well as stability of CARs (40). For example, in first generation CARs, $\mathrm{CD} 3 \zeta$, containing transmembrane domain dimerizes with endogenous TCR and enhances the CAR T cell function as compared to the mutated $\mathrm{CD} 3 \zeta$ transmembrane domain (41). In addition to this Savoldo et al. have shown that CARs containing $\mathrm{CD} 3 \zeta$ transmembrane domain are less stable on the cell surface as compared to the CD28 transmembrane domain in second generation CARs (42). According to Guedan et al. transmembrane domains helps in persistence as well as enhance antitumor efficacy in third generation ICOS based CAR. It was observed that the ICOS TM domain is required for the optimal in vivo phenotype of third generation ICOS based CAR T cells. However, ICOS transmembrane domain did not show any effect on CAR cell-surface expression or tonic signaling (43). Very recently investigators have demonstrated critical role of hinge and transmembrane domain in context of release of effector cytokines which is one of the crucial challenges of CAR T cell therapy known as cytokine release syndrome (CRS) that involves release of excess amounts of cytokines causing toxicity. CAR T cells having $\mathrm{CD} 8 \alpha$ hinge and transmembrane domain release less IFN- $\gamma$ and TNF- $\alpha$ as compared to those having CD28 hinge and transmembrane domain with no significant differences in CAR $\mathrm{T}$ cell efficacy and proliferation (44). Therefore, designing of a robust CAR should involve the best combination of hinge and transmembrane domain to regulate the functionality of CAR T cells.

\section{Regulation by Intracellular Endodomains}

Different generations of CARs vary in their respective intracellular/co-stimulatory signaling domains. While first generation CARs contain only $\mathrm{CD} 3 \zeta$ intracellular domain, the second and third generation CARs contain CD3 $\zeta$ intracellular domain along with either single co-stimulatory domain or two co-stimulatory domains like CD28 and 4-1BB (4).Upon antigen binding phosphorylation cascade of immunoreceptor tyrosinebased activtion motif ITAMs present in CD3 $\zeta$ intracellular domain is initiated leading to activation and priming of CAR T cells (45). However, second generation CAR modification over the first generation CARs by inserting the CD28 co-stimulatory domain demonstrated increased expansion and persistence of CAR T cells as compared to first generation counterparts (42).

On the contrary, 4-1BB (CD137), another co-stimulatory receptor is responsible for enhanced $\mathrm{T}$ cell survival. In a clinical study, the second generation CD19 CAR (CTL019) containing anti-CD19 scFv, CD3 $\zeta$ domain along with 4-1BB co-stimulatory domain revealed robust expansion and long persistence of CTL019 cells along with sustained remissions in patients with relapsed/refractory chronic lymphocytic leukemia CLL (46). Another study using umbilical cord blood $\mathrm{T}$ cells (UCB T cells) revealed that UCB-19BBz and UCB-1928BBz CAR T cells were more cytotoxic toward CD19+ leukemic cell lines as compared to UCB-19z and UCB-1928z CAR T cells (47). Few studies compared the utility of CD28 and $4-1 \mathrm{BB}$ in various CAR constructs and data revealed the almost similar early response rates in ALL patients when treated with either CD28 or $4-1 B B$ CAR $(48,49)$. However, in case of CLL, the 4-1BB CARs exhibited superior efficacy than the CD28 CARs, probably due to increased persistence of 4-1BB (CD137) CAR $\mathrm{T}$ cells and exhaustion of CD28 CAR T cells driven by CD28 endodomain signaling $(46,50,51)$. The study done by Brentjens et al. in 8 CLL patients showed that infusion of $19-28 \mathrm{z} \mathrm{T}$ cells resulted in complete reduction in lymphadenopathy in $1 / 8$ patients (12.5\%), progressive stable disease in $3 / 8$ patients and no objective response in 4 patients. However, according to Porter et al., overall response rate in CLL patients when treated with CTL019 (CD19 scFv+ 4-1BB costimulatory domain) was found to be $8 / 14$ (57\%), where 4 patients showed complete remissions (CR) and 4 showed partial remissions (PR). The CAR T cells persisted and remained functional for more than 4 years in case of two patients who achieved CR and showed no relapse symptoms.

In another study, 4-1BB CARs (GD2-BBz CAR T cells) have been demonstrated to ameliorate CAR $\mathrm{T}$ cell exhaustion by decreasing the expression of exhaustion related molecules and by upregulation of three critical pathways such as hypoxia inducible signaling, cellular metabolism and negative apoptosis regulation (51). However, it remains poorly understood how these pathways contribute in ameliorating CAR T cell exhaustion in 4-1BB CARs.

In addition, CAR signaling impact metabolic reprogramming in $\mathrm{T}$ cells by modulating bioenergetics and mitochondrial biogenesis. The CD28z CAR T signaling facilitates differentiation of $\mathrm{T}_{\mathrm{EM}}$ and increased aerobic glycolysis in $\mathrm{T}$ cells. On the contrary, $4-1 \mathrm{BBz}$ CAR $\mathrm{T}$ cells display differentiation to $\mathrm{T}_{\mathrm{CM}}$ cells along with increased mitochondrial biogenesis and oxidative metabolism (52). The high rate of mitochondrial respiratory capacity as observed in case of $4-1 \mathrm{BBz}$ CAR $\mathrm{T}$ 
cells promotes $\mathrm{CD} 8+\mathrm{T}$ cell memory differentiation and the oxidative phosphorylation acts a major source of energy thereby supporting increased CAR T cell proliferation (53). Therefore, in circumstances where long term CAR T cell persistence can cause severe off tumor toxicity, short-lived CARs can be designed by incorporating CD28 co-stimulatory domains.

Although many studies demonstrated that 4-1BB CAR is safe with increased persistence, in case of $\mathrm{CD} 5+$ tumor targeting, CD5-4-1BB CAR showed reduced efficacy compared with CD5CD28z CAR due to enhanced T cell fratricide. To understand the mechanism of these observations, it has been described that the tumor necrosis factor receptor associated factor (TRAF) signaling induced by 4 -1BB co-stimulatory domains upregulates expression of intracellular adhesion molecule 1 (ICAM-1) (usually not expressed on $\mathrm{T}$ cells) which in turn stabilizes the immunological synapse between CD5 CAR T cells (54). In order to circumvent this CAR (either 4-1BB or others) induced toxicity, a regulated CAR expression system may be developed.

While majority of available CARs either used 4-1BB or CD28 CARs, another CD4-related co-stimulatory receptor OX40 (CD134) emerged as a prominent approach in CAR signaling. Investigators have shown that a third generation CAR CD28z-OX40 helped CCR7 (-) T cells avoid apoptosis and show potent anti-tumor functional efficacy $(55,56)$. Another report by the same group revealed that in comparison to a CD28- $\mathrm{Z}$ CAR, the 3rd generation CD28-z-OX40 CAR decreased secretion of repressive cytokine like IL-10 without altering secretion of pro-inflammatory cytokines, $\mathrm{T}$ cell proliferation and cytotoxic potential. OX40 signaling further repressed Treg mediated IL10 secretion (56). This aspect of OX40 signaling can be harnessed in controlling Treg to effector $\mathrm{T}$ cell ratios in adoptive immunotherapy.

The ICOS expressing CARs induce ICOS signaling in the $\mathrm{T}$ cells and thereby increase the expression of IL-17A, IL-17F, and IL-22 (57). ICOS mediated co-stimulatory CAR signaling established Th17 characteristics such as increased expression of RORC, CD161, IL-1R1, and NCS-1. ICOS signaling also fostered Th17/Th1 polarization by enhancing IFN $-\gamma$ and Tbet expression. In vivo animal studies further revealed enhanced persistence and anti-tumor responses of CD4+ CAR T cells in case of ICOS based CARs in contrast to the CD28 or 4 -1BB containing CARs $(43,57)$. The extended persistence of ICOS-based CARs can be translated to non-lymphoid tumors where CAR T cell persistence is poorly understood. However, effect of ICOS based CARs is yet to be tested clinically in patients.

Hence, the co-stimulatory domains play a key regulatory role in determining the anti-tumor efficacy, functionality and persistence of CAR T cells both in vitro and in vivo. A thorough understanding of the signaling cascades associated with various co-stimulatory molecules will help in designing more effective CAR with better clinical implications.

\section{Regulating CAR T Cell Efficacy With Homeostatic Cytokines and Other Genes}

Efficient $\mathrm{T}$ cell activation requires three signals, $\mathrm{T}$ cell receptor (TCR) signaling (Signal 1), and activation by co-stimulatory molecules (Signal 2) and immune-stimulatory cytokines (Signal 3). So far majority of the CARs designed and discussed possess signal 1 and signal 2, however, signal 3 generally provided by homeostatic cytokines is absent in the conventional CAR T cells and also less abundant in the tumor microenvironment (15) Therefore, next generation of CAR T cells would require an additional cytokine signaling to satisfy the need of signal 3 for optimal CAR $\mathrm{T}$ cell activation (58). The major cytokines involved in $\mathrm{T}$ cell activation belong to $\gamma$ c class like IL-2, IL-7, IL-15, IL-21, and IL-9. These cytokines control T cell survival and proliferation, which ultimately has significant roles in CAR T cell persistence and efficacy (59). These cytokines are currently employed in ex vivo expansion of CAR T cells prior to therapy in combinations or alone (60).

Starting with the well-studied cytokine in $\mathrm{T}$ cell activation and regulation, IL-2 has ranked first upon receiving a US FDA approval for employment in immunotherapy of melanoma in 1998 (61). However, along with induction of potent anti-tumor T cells, IL-2 is also known to cause Activation Induced Cell Death (AICD) and differentiation into immunosuppressive regulatory $\mathrm{T}$ cells (62). Hence, low dose IL-2 in CAR T cell therapy has been suggested for achieving better anti-tumor responses and to overcome the immunosuppressive effects of IL-2 (63).

Another approach to lessen the immunosuppressive effect of IL-2 on T lymphocyte is to make way for other cytokines like IL-7 and IL-15 in adoptive immunotherapy. With the discovery of T stem cell like memory cells $\left(\mathrm{T}_{\mathrm{scm}}\right)$ it has been described that IL-7 and IL-15 are known to generate and maintain less differentiated $\mathrm{T}_{\text {SCM }}$ population in the $\mathrm{T}$ lymphocyte pool thereby improving the antitumor responses significantly (64-66). In the absence of these regulatory cytokines the differentiation status of CAR T cells is skewed toward terminal differentiation thereby reducing functional potency of the CAR T cells. Owing to this fact, incorporation of IL-7 and IL-15 in the in vivo expansion of CAR $\mathrm{T}$ cells has been a new addition in the adoptive immunotherapy field (60).

Attempts have been made for administration of cytokines like IL-2, IL-7, IL-15, and IL-12 in clinical trials against various malignancies. However, the anti-tumor responses are greatly masked by toxicities generated due to the cytokine administration alone or along with CAR T cells (67-70). The toxic effects of recombinant cytokines are due to higher doses of i.v. administration in patients leading to off target toxicity. Therefore, attempts are been made to engineer CAR $\mathrm{T}$ cells to secrete or express these cytokines on the $\mathrm{T}$ cell surface giving rise to next generation of CAR T cells. A variant of IL-7R has been engineered to be expressed along with CAR T cells against solid tumors and has shown a good response in improving the anti-tumor efficacy in vivo models (71). CAR $\mathrm{T}$ cells secreting IL-15 have been tested in hematological malignancies along with anti-CD19 CAR expression. The IL-15 expression has shown to regulate differentiation of these modified CAR $\mathrm{T}$ cells by inhibiting apoptosis and showing reduced Treg induction unlike IL-2 (72). Along with the secretory form, the membrane bound expression of IL-15 has also shown to be a good strategy in achieving less differentiated CAR T cells and in generating potent anti-tumor responses in leukemia models (73). 
In addition to this, IL-12 armored CAR T cells has shown to eliminate the need of pre-treatment regime before the onset of CAR T therapy in a preclinical setting. IL-12 armored CAR $\mathrm{T}$ cells also possess an intrinsic resistance to regulatory $\mathrm{T}$ cells mediated inhibition (74). However, another group of researchers conducting clinical trials with engineered TILs to secrete IL12 showed a highly toxic response owing to the uncontrolled cytokine release (75). In addition, a report suggested leukemic transformation and clonal expansion of CD8 $+\mathrm{T}$ cells transduced to express IL-15 gene. This further helped in realizing the need for control of signal 3 generated by immune regulatory cytokines (76).

In order to regulate the adverse effects mediated by cytokine secretion as well as to regulate toxicities due to CAR T cells as observed in clinical trials, the scientists are designing strategies to tune these cells in a ligand dependent manner (77). One such "safety-switch" is the inducible caspase 9 molecule integrated with CAR T cells which can be controlled by an external ligand AP1903. Administration of the ligand induces caspase 9 mediated apoptotic pathway thereby clearing the CAR T cells from body and reducing the off-target cytotoxicity. These switches have been combined with cytokines IL-7 and IL-15 in the preclinical studies to provide a hope for managing toxicities due to the uncontrolled cytokine secretion $(71,78)$.

With such newer modifications in the CAR T cell designs, the focus of the field is gradually shifting toward regulation of actions of CAR T cells with presence of tunable signals and use of inhibitory receptors. Very recently, a new generation of CAR T cells, the SUPRA CAR T cells is been demonstrated (79). SUPRA stands for Split, Universal and Programmable CAR T cells, which can respond to multiple antigens and can be inducible controlled to manage $\mathrm{T}$ cell activation mediated toxicity. Other such CAR T cells such as iCARs (inhibitory CARs) are analyzed in preclinical studies wherein use of CTLA-4 and PD-1 based self-regulating inhibitory receptors have been employed to control off-target toxicities (80).

The current advances in the employment of cytokines and receptors thereof in regulating the CAR cell response is more on the preclinical front. The clinical outputs of these engineered CAR T cells along with newly designed switches would provide a useful insight into planning strategies for better regulation of CAR T cells in immunotherapy. Until then, the management of signal 3 for the regulation of CAR T cells stills remains elusive and opens a new field of research in adoptive immunotherapy.

\section{CONCLUSION AND FUTURE PROSPECTIVE}

Chimeric antigen receptors emerged as new genre of drugs with huge therapeutic potential for hematological malignancies as well as solid tumors. Different components of a CAR strongly impact the anti-tumor efficacy, potency and safety of the genetically engineered CAR T cells after infusion into the patients. This review highlights the importance of $\mathrm{scFv}$ designing in determining flexibility, affinity and specificity of the CAR along with strategies to minimize the off target toxicity. The regulatory role of hinge domain and transmembrane domain in providing proper positioning of the CAR, imparting stability and reducing cytokine release toxicity has also been discussed. Moreover, this review conveys the key role of intracellular domain in relation to long term survival and persistence of CAR T cells along with the regulatory aspects of various cytokines like IL-2, IL-15, IL-7, and IL-21. Fine tuning of the crucial components and parameters as discussed in this review will pave the way for developing more safe and efficacious CAR T cells for a wide array of malignancies.

Despite the successful achievements of CAR T cell therapy in case of hematological malignancies it displays to serious side effects such as cytokine release syndrome (CRS) that causes systemic inflammatory responses, multi-organ failure and death as well as neuro-toxic effects like aphasia, hallucinations and seizures (67). Another major concern is the "on target off-tumor" toxicity. The efficacy and safety of CAR T cells is monitored in preclinical animal models prior to its clinical testing, which includes syngeneic, human xenograft, immunocompetent transgenic, and humanized transgenic mice. These pre-clinical models neither reflect the obstacles in clinical efficacy nor do they predict potentially life-threatening safety concerns and hence lack to display the complications which might arise in the clinical setting. Moreover, the lack of host immune system does not allow testing of the tumor microenvironment, the tumor's metastatic potential, or the host response to CAR T cells. Each murine model has its own advantages and shortcomings which highlights the absence of a single competent model that can evaluate CAR $\mathrm{T}$ cell efficacy as well as toxicity issues. Recently, many primate models such as macaque or dog are also being studied in relation to CAR T side effects $(81,82)$. Hence, the evolution and refinement of preclinical models will lead to improved prediction of CAR T safety and efficacy in the clinic.

With all the evidences available with varied designs of the CAR in the field, so far it remains elusive to understand the use of correct combination of these domains which regulate the CAR T cell efficacy. In order to generate a predictive model to understand the efficacy and toxicity of the designed CAR T cells in in vitro experiments, arises the need to design computational biology tools. The development of computational approaches with a combination of clinical outcomes from the currently developed CAR T cells, may have a major impact in the designing superior CAR T cells with minimal toxicity and improved efficacy in future.

\section{AUTHOR CONTRIBUTIONS}

All authors listed have made a substantial, direct and intellectual contribution to the work, and approved it for publication.

\section{FUNDING}

This work was supported by intramural grants of IIT Bombay (15IRSGHC002), funding from Wadhwani research foundation (DO/2017-WRCB002-016/PRE_APPRVL_REQ/188581), Tata center (DGDON422/PRE_APPRVL_REQ/147655) at IIT Bombay and Tata Trust (RD/0117-TATAE00-001), to RP. 


\section{REFERENCES}

1. Miliotou AN, Papadopoulou LC. CAR T-cell Therapy: a new era in cancer immunotherapy. Curr Pharm Biotechnol. (2018) 19:5-18. doi: 10.2174/1389201019666180418095526

2. Grupp SA, Kalos M, Barrett D, Aplenc R, Porter DL, Rheingold SR, et al. Chimeric antigen receptor-modified T cells for acute lymphoid leukemia. $N$ Engl J Med. (2013) 368:1509-18. doi: 10.1056/NEJMoa1215134

3. Kalos M, Levine BL, Porter DL, Katz S, Grupp SA, Bagg A, et al. T cells with chimeric antigen receptors have potent antitumor effects and can establish memory in patients with advanced leukemia. Sci Transl Med. (2011) 3:95ra73. doi: 10.1126/scitranslmed.3002842

4. Maus MV, Grupp SA, Porter DL, June CH. Antibody-modified T cells: CARs take the front seat for hematologic malignancies. Blood (2014) 123:2625-35. doi: 10.1182/blood-2013-11-492231

5. Li J, Li W, Huang K, Zhang Y, Kupfer G, Zhao Q. Chimeric antigen receptor $\mathrm{T}$ cell (CAR-T) immunotherapy for solid tumors: lessons learned and strategies for moving forward. J Hematol Oncol. (2018) 11:22. doi: 10.1186/s13045-018-0568-6

6. Chailyan A, Marcatili P, Tramontano A. The association of heavy and light chain variable domains in antibodies: implications for antigen specificity. FEBS J. (2011) 278:2858-66. doi: 10.1111/j.1742-4658.2011.08207.x

7. Lonberg N. Fully human antibodies from transgenic mouse and phage display platforms. Curr Opin Immunol. (2008) 20:450-9. doi: 10.1016/j.coi.2008.06.004

8. Golynskiy MV, Haugner JC, Morelli A, Morrone D, Seelig B. In vitro evolution of enzymes In: Samuelson JC, editor. Enzyme Engineering. Totowa, NJ: Humana Press (2013). p. 73-92. doi: 10.1007/978-1-62703-293-3_6

9. $\mathrm{Lu} \mathrm{W}-\mathrm{C}$, Ellington $\mathrm{AD}$. In vitro selection of proteins via emulsion compartments. Methods (2013) 60:75-80. doi: 10.1016/j.ymeth.2012.03.008

10. Zahnd C, Amstutz P, Plückthun A. Ribosome display: selecting and evolving proteins in vitro that specifically bind to a target. Nat Methods (2007) 4:26979. doi: $10.1038 /$ nmeth1003

11. Hoogenboom HR. Selecting and screening recombinant antibody libraries. Nat Biotechnol. (2005) 23:1105-16. doi: 10.1038/nbt1126

12. Doerner A, Rhiel L, Zielonka S, Kolmar H. Therapeutic antibody engineering by high efficiency cell screening. FEBS Lett. (2014) 588:278-87. doi: 10.1016/j.febslet.2013.11.025

13. Wittrup KD. Protein engineering by cell-surface display. Curr Opin Biotechnol. (2001) 12:395-9. doi: 10.1016/S0958-1669(00)00233-0

14. Ahmad ZA, Yeap SK, Ali AM, Ho WY, Alitheen NBM, Hamid M. scFv antibody: principles and clinical application. Clin Dev Immunol. (2012) 2012:980250. doi: 10.1155/2012/980250

15. Kershaw MH, Westwood JA, Darcy PK. Gene-engineered T cells for cancer therapy. Nat Rev Cancer (2013) 13:525-41. doi: 10.1038/nrc3565

16. Maus MV, Haas AR, Beatty GL, Albelda SM, Levine BL, Liu X, et al. $\mathrm{T}$ cells expressing chimeric antigen receptors can cause anaphylaxis in humans. Cancer Immunol Res. (2013) 1:26-31. doi: 10.1158/2326-6066.CIR13-0006

17. Miotti S, Canevari S, Menard S, Mezzanzanica D, Porro G, Pupa SM, et al. Characterization of human ovarian carcinoma-associated antigens defined by novel monoclonal antibodies with tumor-restricted specificity. Int J Cancer (1987) 39:297-303. doi: 10.1002/ijc.2910390306

18. O’Rourke DM, Nasrallah MP, Desai A, Melenhorst JJ, Mansfield K, Morrissette JJD, et al. A single dose of peripherally infused EGFRvIIIdirected CAR $\mathrm{T}$ cells mediates antigen loss and induces adaptive resistance in patients with recurrent glioblastoma. Sci Transl Med. (2017) 9:eaaa0984. doi: 10.1126/scitranslmed.aaa0984

19. Adusumilli PS, Cherkassky L, Villena-Vargas J, Colovos C, Servais E, Plotkin $\mathrm{J}$, et al. Regional delivery of mesothelin-targeted CAR T cell therapy generates potent and long-lasting CD4-dependent tumor immunity. Sci Transl Med. (2014) 6:261ra151. doi: 10.1126/scitranslmed.3010162

20. Fry TJ, Shah NN, Orentas RJ, Stetler-Stevenson M, Yuan CM, Ramakrishna S, et al. CD22-targeted CAR T cells induce remission in B-ALL that is naive or resistant to CD19-targeted CAR immunotherapy. Nat Med. (2018) 24:20-8. doi: $10.1038 / \mathrm{nm} .4441$

21. Kershaw MH, Westwood JA, Parker LL, Wang G, Eshhar Z, Mavroukakis SA, et al. A phase I study on adoptive immunotherapy using gene-modified
$\mathrm{T}$ cells for ovarian cancer. Clin Cancer Res. (2006) 12:6106-15. doi: 10.1158/1078-0432.CCR-06-1183

22. Morgan RA, Yang JC, Kitano M, Dudley ME, Laurencot CM, Rosenberg SA. Case report of a serious adverse event following the administration of $\mathrm{T}$ cells transduced with a chimeric antigen receptor recognizing ERBB2. Molecular Ther. (2010) 18:843-51. doi: 10.1038/mt.2010.24

23. Ahmed N, Brawley VS, Hegde M, Robertson C, Ghazi A, Gerken C, et al. Human epidermal growth factor receptor 2 (HER2) -specific chimeric antigen receptor-modified T cells for the immunotherapy of HER2-positive sarcoma. J Clin Oncol. (2015) 33:1688-96. doi: 10.1200/JCO.2014.58.0225

24. Hudecek M, Lupo-Stanghellini MT, Kosasih PL, Sommermeyer D, Jensen MC, Rader C, et al. Receptor affinity and extracellular domain modifications affect tumor recognition by ROR1-specific chimeric antigen receptor T cells. Clin Cancer Res. (2013) 19:3153-64. doi: 10.1158/1078-0432.CCR-13-0330

25. Lynn RC, Feng Y, Schutsky K, Poussin M, Kalota A, Dimitrov DS, et al. High-affinity FRbeta-specific CAR $\mathrm{T}$ cells eradicate AML and normal myeloid lineage without HSC toxicity. Leukemia (2016) 30:1355-64. doi: 10.1038/leu.2016.35

26. Liu X, Jiang S, Fang C, Yang S, Olalere D, Pequignot EC, et al. AffinityTuned ErbB2 or EGFR chimeric antigen receptor T cells exhibit an increased therapeutic index against tumors in mice. Cancer Res. (2015) 75:3596-607. doi: 10.1158/0008-5472.CAN-15-0159

27. James SE, Greenberg PD, Jensen MC, Lin Y, Wang J, Till BG, et al. Antigen sensitivity of CD22-specific chimeric TCR is modulated by target epitope distance from the cell membrane. J Immunol. (2008) 180:7028-38. doi: 10.4049/jimmunol.180.10.7028

28. Lee DW, Stetler-Stevenson M, Yuan CM, Shah NN, Delbrook C, Yates B, et al. Long-term outcomes following CD19 CAR T cell therapy for B-ALL are superior in patients receiving a fludarabine/cyclophosphamide preparative regimen and post-CAR hematopoietic stem cell transplantation. Am Soc Hematol. (2016) 128:218

29. Maude SL, Laetsch TW, Buechner J, Rives S, Boyer M, Bittencourt H, et al. Tisagenlecleucel in children and young adults with B-cell lymphoblastic leukemia. N Engl J Med. (2018) 378:439-48. doi: 10.1056/NEJMoa1709866

30. Park JH, Rivière I, Gonen M, Wang X, Sénéchal B, Curran KJ, et al. Long-term follow-up of CD19 CAR therapy in acute lymphoblastic leukemia. $N$ Engl J Med. (2018) 378:449-59. doi: 10.1056/NEJMoa1709919

31. Larson RP, Ports M, Jessup H, Manriquez G, Hay C, Stewart R, et al. Preclinical analyses support clinical investigation of combined PD-L1 blockade and antiCD19 CAR T cell therapy for the treatment of NHL. Am Soc Hematol. (2015) 126:5430.

32. Mackall CL. Enhancing the efficacy of CAR T cells. Am Soc Hematol. (2017) 130:SCI-15.

33. Schneider D, Xiong Y, Wu D, Nölle V, Schmitz S, Haso W, et al. A tandem CD19/CD20 CAR lentiviral vector drives on-target and off-target antigen modulation in leukemia cell lines. J Immunother Cancer (2017) 5:42. doi: 10.1186/s40425-017-0246-1

34. Zah E, Lin, M.-Y., Silva-Benedict A, Jensen MC, and Chen YY. T cells expressing CD19/CD20 bi-specific chimeric antigen receptors prevent antigen escape by malignant B cells. Cancer Immunol Res. (2016) 4:498-508. doi: 10.1158/2326-6066.CIR-15-0231

35. Hegde M, Mukherjee M, Grada Z, Pignata A, Landi D, Navai SA, et al. Tandem CAR T cells targeting HER2 and IL13R $\alpha 2$ mitigate tumor antigen escape. $J$ Clin Invest. (2016) 126:3036-52. doi: 10.1172/JCI83416

36. Guest RD, Hawkins RE, Kirillova N, Cheadle EJ, Arnold J, O'Neill A, et al. The role of extracellular spacer regions in the optimal design of chimeric immune receptors: evaluation of four different scFvs and antigens. J Immunother. (2005) 28:203-11. doi: 10.1097/01.cji.0000161397.96582.59

37. Hudecek M, Sommermeyer D, Kosasih PL, Silva-Benedict A, Liu L, Rader $\mathrm{C}$, et al. The nonsignaling extracellular spacer domain of chimeric antigen receptors is decisive for in vivo antitumor activity. Cancer Immunol Res. (2015) 3:125-35. doi: 10.1158/2326-6066.CIR-14-0127

38. Qin L, Lai Y, Zhao R, Wei X, Weng J, Lai P, et al. Incorporation of a hinge domain improves the expansion of chimeric antigen receptor $\mathrm{T}$ cells. J Hematol Oncol. (2017) 10:68. doi: 10.1186/s13045-017-0437-8

39. Watanabe N, Bajgain P, Sukumaran S, Ansari S, Heslop HE, Rooney CM, et al. Fine-tuning the CAR spacer improves T-cell potency. Oncoimmunology (2016) 5:e1253656. doi: 10.1080/2162402X.2016.1253656 
40. Shirasu N, Kuroki M. Functional design of chimeric T-cell antigen receptors for adoptive immunotherapy of cancer: architecture and outcomes. Anticancer Res. (2012) 32:2377-83.

41. Bridgeman JS, Hawkins RE, Bagley S, Blaylock M, Holland M, Gilham DE. The optimal antigen response of chimeric antigen receptors harboring the CD3zeta transmembrane domain is dependent upon incorporation of the receptor into the endogenous TCR/CD3 complex. J Immunol. (2010) 184:6938-49. doi: 10.4049/jimmunol.0901766

42. Savoldo B, Ramos CA, Liu E, Mims MP, Keating MJ, Carrum G, et al. CD28 costimulation improves expansion and persistence of chimeric antigen receptor-modified T cells in lymphoma patients. J Clin Invest. (2011) 121:1822-6. doi: 10.1172/JCI46110

43. Guedan S, Posey, A. D. Jr., Shaw C, Wing A, Da T, Patel PR, et al. Enhancing CAR T cell persistence through ICOS and 4-1BB costimulation. JCI Insight (2018) 3:96976. doi: 10.1172/jci.insight.96976

44. Alabanza L, Pegues M, Geldres C, Shi V, Wiltzius JJW, Sievers SA, et al. Function of novel anti-CD19 chimeric antigen receptors with human variable regions is affected by hinge and transmembrane domains. Mol Ther. (2017) 25:2452-65. doi: 10.1016/j.ymthe.2017.07.013

45. Dotti G, Gottschalk S, Savoldo B, Brenner MK. Design and development of therapies using chimeric antigen receptor-expressing T cells. Immunol Rev. (2014) 257:107-26. doi: 10.1111/imr.12131

46. Porter DL, Hwang WT, Frey NV, Lacey SF, Shaw PA, Loren AW, et al. Chimeric antigen receptor $\mathrm{T}$ cells persist and induce sustained remissions in relapsed refractory chronic lymphocytic leukemia. Sci Transl Med. (2015) 7:303ra139. doi: 10.1126/scitranslmed.aac5415

47. Tammana S, Huang X, Wong M, Milone MC, Ma L, Levine BL, et al. 4$1 \mathrm{BB}$ and CD28 signaling plays a synergistic role in redirecting umbilical cord blood T cells against B-cell malignancies. Hum Gene Ther. (2010) 21:75-86. doi: 10.1089/hum.2009.122

48. Brentjens RJ, Davila ML, Riviere I, Park J, Wang X, Cowell LG, et al. CD19-targeted $\mathrm{T}$ cells rapidly induce molecular remissions in adults with chemotherapy-refractory acute lymphoblastic leukemia. Sci Transl Med. (2013) 5:177ra138. doi: 10.1126/scitranslmed.3005930

49. Lee DW, Kochenderfer JN, Stetler-Stevenson M, Cui YK, Delbrook C, Feldman SA, et al. T cells expressing CD19 chimeric antigen receptors for acute lymphoblastic leukaemia in children and young adults: a phase 1 dose-escalation trial. Lancet (2015) 385:517-28. doi: 10.1016/S0140-6736(14)61403-3

50. Brentjens RJ, Riviere I, Park JH, Davila ML, Wang X, Stefanski J, et al. Safety and persistence of adoptively transferred autologous CD19-targeted T cells in patients with relapsed or chemotherapy refractory B-cell leukemias. Blood (2011) 118:4817-28. doi: 10.1182/blood-2011-04-348540

51. Long AH, Haso WM, Shern JF, Wanhainen KM, Murgai M, Ingaramo $\mathrm{M}$, et al. 4-1BB costimulation ameliorates $\mathrm{T}$ cell exhaustion induced by tonic signaling of chimeric antigen receptors. Nat Med. (2015) 21:581-90. doi: $10.1038 / \mathrm{nm} .3838$

52. Kawalekar OU, RS OC, Fraietta JA, Guo L, McGettigan SE, Posey ADJr, et al. Distinct signaling of coreceptors regulates specific metabolism pathways and impacts memory development in CAR T cells. Immunity (2016) 44:712. doi: 10.1016/j.immuni.2016.02.023

53. van der Windt GJ, O'Sullivan D, Everts B, Huang SC, Buck MD, Curtis JD, et al. CD8 memory T cells have a bioenergetic advantage that underlies their rapid recall ability. Proc Natl Acad Sci USA. (2013) 110:14336-41. doi: 10.1073/pnas.1221740110

54. Mamonkin M, Mukherjee M, Srinivasan M, Sharma S, Gomes-Silva D, Mo F, et al. Reversible transgene expression reduces fratricide and permits 4-1BB costimulation of CAR T cells directed to T-cell malignancies. Cancer Immunol Res. (2018) 6:47-58. doi: 10.1158/2326-6066.CIR-17-0126

55. Hombach AA, Chmielewski M, Rappl G, Abken H. Adoptive immunotherapy with redirected $\mathrm{T}$ cells produces CCR7- cells that are trapped in the periphery and benefit from combined CD28-OX40 costimulation. Hum Gene Ther. (2013) 24:259-69. doi: 10.1089/hum.2012.247

56. Hombach AA, Heiders J, Foppe M, Chmielewski M, Abken H. OX40 costimulation by a chimeric antigen receptor abrogates CD28 and IL-2 induced IL-10 secretion by redirected CD4(+) T cells. Oncoimmunology (2012) 1:458-66.
57. Guedan S, Chen X, Madar A, Carpenito C, McGettigan SE, Frigault MJ, et al. ICOS-based chimeric antigen receptors program bipolar TH17/TH1 cells. Blood (2014) 124:1070-80. doi: 10.1182/blood-2013-10-535245

58. Minton K. Immunotherapy: cytokine boost for CAR T cells. Nat Rev Immunol. (2018) 18:150-1. doi: 10.1038/nri.2018.8

59. Xu XJ, Song DG, Poussin M, Ye Q, Sharma P, Rodriguez-Garcia A, et al. Multiparameter comparative analysis reveals differential impacts of various cytokines on CART cell phenotype and function ex vivo and in vivo. Oncotarget (2016) 7:82354-68. doi: 10.18632/oncotarget.10510

60. Xu Y, Zhang M, Ramos CA, Durett A, Liu E, Dakhova O, et al. Closely related T-memory stem cells correlate with in vivo expansion of CAR.CD19$\mathrm{T}$ cells and are preserved by IL-7 and IL-15. Blood (2014) 123:3750-9. doi: 10.1182/blood-2014-01-552174

61. Rosenberg SA. IL-2: the first effective immunotherapy for human cancer. J Immunol. (2014) 192:5451-8. doi: 10.4049/jimmunol.1490019

62. Hartmann J, Schussler-Lenz M, Bondanza A, Buchholz CJ. Clinical development of CAR $\mathrm{T}$ cells-challenges and opportunities in translating innovative treatment concepts. EMBO Mol Med. (2017) 9:1183-97. doi: 10.15252/emmm.201607485

63. Kaartinen T, Luostarinen A, Maliniemi P, Keto J, Arvas M, Belt H, et al. Low interleukin-2 concentration favors generation of early memory $\mathrm{T}$ cells over effector phenotypes during chimeric antigen receptor T-cell expansion. Cytotherapy (2017) 19:1130. doi: 10.1016/j.jcyt.2017.06.003

64. Busch DH, Frassle SP, Sommermeyer D, Buchholz VR, Riddell SR. Role of memory $\mathrm{T}$ cell subsets for adoptive immunotherapy. Semin Immunol. (2016) 28:28-34. doi: 10.1016/j.smim.2016.02.001

65. Cieri N, Camisa B, Cocchiarella F, Forcato M, Oliveira G, Provasi E, et al. IL-7 and IL-15 instruct the generation of human memory stem T cells from naive precursors. Blood (2013) 121:573-84. doi: 10.1182/blood-2012-05-431718

66. Gattinoni L, Lugli E, Ji Y, Pos Z, Paulos CM, Quigley MF, et al. A human memory T cell subset with stem cell-like properties. Nat Med. (2011) 17:12907. doi: $10.1038 / \mathrm{nm} .2446$

67. Brudno JN, Kochenderfer JN. Toxicities of chimeric antigen receptor $\mathrm{T}$ cells: recognition and management. Blood (2016) 127:3321-30. doi: 10.1182/blood-2016-04-703751

68. Conlon KC, Lugli E, Welles HC, Rosenberg SA, Fojo AT, Morris JC, et al. Redistribution, hyperproliferation, activation of natural killer cells and CD8 T cells, and cytokine production during first-in-human clinical trial of recombinant human interleukin-15 in patients with cancer. J Clin Oncol. (2015) 33:74-82. doi: 10.1200/JCO.2014.57.3329

69. Schwartz RN, Stover L, Dutcher JP. Managing toxicities of high-dose interleukin-2. Oncology (2002) 16:11-20.

70. Sportes C, Babb RR, Krumlauf MC, Hakim FT, Steinberg SM, Chow CK, et al. Phase I study of recombinant human interleukin-7 administration in subjects with refractory malignancy. Clin Cancer Res. (2010) 16:727-35. doi: 10.1158/1078-0432.CCR-09-1303

71. Shum T, Omer B, Tashiro H, Kruse RL, Wagner DL, Parikh K, et al. Constitutive Signaling from an Engineered IL7 Receptor Promotes Durable Tumor Elimination by Tumor-Redirected T Cells. Cancer Discov. (2017) 7:1238-47. doi: 10.1158/2159-8290.CD-17-0538

72. Hsu C, Hughes MS, Zheng Z, Bray RB, Rosenberg SA, Morgan RA. Primary human $\mathrm{T}$ lymphocytes engineered with a codon-optimized IL-15 gene resist cytokine withdrawal-induced apoptosis and persist long-term in the absence of exogenous cytokine. J Immunol. (2005) 175:7226-34. doi: 10.4049/jimmunol.175.11.7226

73. Hurton LV, Singh H, Najjar AM, Switzer KC, Mi T, Maiti S, et al. Tethered IL-15 augments antitumor activity and promotes a stem-cell memory subset in tumor-specific T cells. Proc Natl Acad Sci USA. (2016) 113:E7788-97. doi: 10.1073/pnas.1610544113

74. Pegram HJ, Lee JC, Hayman EG, Imperato GH, Tedder TF, Sadelain M, et al. Tumor-targeted $\mathrm{T}$ cells modified to secrete IL-12 eradicate systemic tumors without need for prior conditioning. Blood (2012) 119:4133-41. doi: 10.1182/blood-2011-12-400044

75. Zhang L, Morgan RA, Beane JD, Zheng Z, Dudley ME, Kassim SH, et al. Tumor-infiltrating lymphocytes genetically engineered with an inducible gene encoding interleukin-12 for the immunotherapy of metastatic melanoma. Clin Cancer Res. (2015) 21:2278-88. doi: 10.1158/1078-0432.CCR-14-2085 
76. Hsu C, Jones SA, Cohen CJ, Zheng Z, Kerstann K, Zhou J, et al. Cytokineindependent growth and clonal expansion of a primary human CD8+ T-cell clone following retroviral transduction with the IL-15 gene. Blood (2007) 109:5168-77. doi: 10.1182/blood-2006-06-029173

77. Gargett T, Brown MP. The inducible caspase-9 suicide gene system as a "safety switch" to limit on-target, off-tumor toxicities of chimeric antigen receptor T cells. Front Pharmacol. (2014) 5:235. doi: 10.3389/fphar.2014. 00235

78. Hoyos V, Savoldo B, Quintarelli C, Mahendravada A, Zhang M, Vera J, et al. Engineering CD19-specific T lymphocytes with interleukin-15 and a suicide gene to enhance their anti-lymphoma/leukemia effects and safety. Leukemia (2010) 24:1160-70. doi: 10.1038/leu.2010.75

79. Cho JH, Collins JJ, and Wong WW. Universal chimeric antigen receptors for multiplexed and logical control of T cell responses. Cell (2018) 173:1426-38 e1411. doi: 10.1016/j.cell.2018.03.038

80. Fedorov VD, Themeli M, and Sadelain M. (2013). PD-1- and CTLA-4-based inhibitory chimeric antigen receptors (iCARs) divert off-target immunotherapy responses. Sci Transl Med. 5:215ra172. doi: 10.1126/scitranslmed.3006597
81. Dong L, Gill S, Bhoj V, Shestova O, Martin L, Aibo DI, et al. 399. Evaluation of CD123 targeting CART Cells in non-human primates. Mol Ther. 24:S158. doi: 10.1016/S1525-0016(16)33208-7

82. Panjwani MK, Smith JB, Schutsky K, Gnanandarajah J, O’Connor CM, Powell DJJr, et al. Feasibility and safety of RNA-transfected CD20-specific chimeric antigen receptor T cells in dogs with spontaneous B cell lymphoma. Mol Ther. (2016) 24:1602-14. doi: 10.1038/mt.2016.146

Conflict of Interest Statement: The authors declare that the research was conducted in the absence of any commercial or financial relationships that could be construed as a potential conflict of interest.

Copyright (c) 2019 Dwivedi, Karulkar, Ghosh, Rafiq and Purwar. This is an openaccess article distributed under the terms of the Creative Commons Attribution License (CC BY). The use, distribution or reproduction in other forums is permitted, provided the original author(s) and the copyright owner(s) are credited and that the original publication in this journal is cited, in accordance with accepted academic practice. No use, distribution or reproduction is permitted which does not comply with these terms. 